PROCEEDINGS OF THE

AMERICAN MATHEMATICAL SOCIETY

Volume 129, Number 7, Pages 2061-2067

S 0002-9939(00)05996-7

Article electronically published on December 4, 2000

\title{
GLOBAL ANALYTIC AND GEVREY HYPOELLIPTICITY OF SUBLAPLACIANS UNDER DIOPHANTINE CONDITIONS
}

\author{
A. ALEXANDROU HIMONAS
}

(Communicated by David S. Tartakoff)

\begin{abstract}
In this paper we consider the problem of global Gevrey and analytic regularity for a class of partial differential operators on a torus in the form of a sum of squares of vector fields, which may not satisfy the bracket condition. We show that these operators are globally Gevrey or analytic hypoelliptic on the torus if and only if the coefficients satisfy certain Diophantine approximation properties.
\end{abstract}

\section{INTRODUCTION AND RESUlT}

On a torus and more generally on an analytic manifold the characterization of the analytic operators, written as a sum of squares of vector fields, sublaplacians, which are globally analytic, or Gevrey hypoelliptic, is an open problem. The motivation of this work is to show that some of the operators considered in [CH] can be analytic or Gevrey hypoelliptic under a condition weaker than the bracket condition. For stating this condition and our result, we need the following definitions.

Let $U$ be an open subset of $\mathbb{R}^{N}$, and $s \geq 1$. A distribution $u \in \mathcal{D}^{\prime}(U)$ is said to belong to the Gevrey class $G^{s}$ at $x_{0} \in U$ if there exists a neighborhood $V \subset U$ of $x_{0}$ such that $u \in C^{\infty}(V)$, and a constant $C>0$ such that

$$
\left|\partial^{\alpha} u(x)\right| \leq C^{|\alpha|+1}|\alpha|^{s \alpha}, \forall x \in V, \forall \alpha \in \mathbb{N}^{N} .
$$

If $u$ is in $G^{s}$ at every point $x_{0} \in U$, then $u$ is said to belong in $G^{s}(U)$. It can be shown (see for example Rodino [R]) that $u \in \mathcal{D}^{\prime}(U)$ is in $G^{s}$ at $x_{0} \in U$ if, and only if, there is a distribution $v$ with compact support agreeing with $u$ near $x_{0}$ and such that its Fourier transform satisfies the estimate

$$
|\hat{v}(\xi)| \leq C e^{-\varepsilon|\xi|^{\frac{1}{s}}}, \forall \xi \in \mathbb{R}^{N}
$$

for some $\varepsilon>0$ and $C>0$.

Let $\mathbb{T}^{N}$ denote the $N$-dimensional torus. Then a distribution $u \in \mathcal{D}^{\prime}\left(\mathbb{T}^{N}\right)$ is in $G^{s}\left(\mathbb{T}^{N}\right)$ if and only if

$$
|\hat{u}(\xi)| \leq C e^{-\varepsilon|\xi|^{\frac{1}{s}}}, \forall \xi \in \mathbb{Z}^{N}
$$

Received by the editors November 15, 1999.

1991 Mathematics Subject Classification. Primary 35H05.

Key words and phrases. Global, analytic and Gevrey hypoellipticity, Diophantine conditions, torus, Fourier transform, bracket condition.

The author was supported in part by NSF Grant DMS-9970857. 
for some $\varepsilon>0$ and $C>0$. A linear partial differential operator $P$ defined on $\mathbb{T}^{N}$ is said to be globally $G^{s}$ hypoelliptic on $\mathbb{T}^{N}$ if for any $u \in \mathcal{D}^{\prime}\left(\mathbb{T}^{N}\right)$ the condition $P u \in G^{s}\left(\mathbb{T}^{N}\right)$ implies that $u \in G^{s}\left(\mathbb{T}^{N}\right)$. If $P$ is defined on an open set $U$ of $\mathbb{R}^{N}$, then $P$ is said to be locally $G^{s}$ hypoelliptic if for any $V$ open subset of $U$ and any $u \in \mathcal{D}^{\prime}(V)$ the condition $P u \in G^{s}(V)$ implies that $u$ is in $G^{s}(V)$. Note that local $G^{s}$ hypoellipticity implies global. Similar are the definitions for $C^{\infty}$ hypoellipticity.

Also we shall need the following number theory definitions.

Definition 1.1. Let $s \geq 1$. A collection of vectors $v_{1}, \ldots, v_{\ell}$ in $\mathbb{R}^{d}$ are not simultaneously approximable with exponent $s$ if for any $\varepsilon>0$ there is $C_{\varepsilon}>0$ such that for each $\eta=\left(\eta_{1}, \ldots, \eta_{\ell}\right) \in \mathbb{Z}^{\ell}$ and each $\xi \in \mathbb{Z}^{d}-0$ we have

$$
\left|\eta_{j}-v_{j} \cdot \xi\right| \geq C_{\varepsilon} e^{-\varepsilon|\xi|^{\frac{1}{s}}} \text {, for some } j=1, \ldots, \ell .
$$

If $\ell=d=s=1$, then this is the definition of a not exponentially Liouville number. It follows that $v_{1}, \ldots, v_{\ell}$ are simultaneously approximable with exponent $s$ if and only if there is $\varepsilon>0$ for which there exist two sequences $\left\{\eta_{k}\right\}=\left\{\left(\eta_{k, 1}, \ldots, \eta_{k, \ell}\right)\right\} \subset \mathbb{Z}^{\ell}$ and $\left\{\xi_{k}\right\}=\left\{\left(\xi_{k, 1}, \ldots, \xi_{k, d}\right)\right\} \subset \mathbb{Z}^{d}$ such that

$$
\left|\eta_{k, j}-v_{j} \cdot \xi_{k}\right| \leq e^{-\varepsilon\left|\xi_{k}\right|^{\frac{1}{s}}}, \forall j=1, \ldots, \ell .
$$

Definition 1.2. Let $\left(b_{1}(t), \ldots, b_{\ell}(t)\right)$ be a vector of real-valued functions which are linearly independent over $\mathbb{R}$. A vector of functions $\left(f_{1}(t), \ldots, f_{d}(t)\right)$ is said to be not simultaneously approximable with exponent $s$ for the basis $\left(b_{1}(t), \ldots, b_{\ell}(t)\right)$, and use the notation $\left(f_{1}, \ldots, f_{d}\right) \in(S A)_{s}^{c}\left(b_{1}, \ldots, b_{\ell}\right)$, if the following two conditions hold:

(1) $\left\{f_{1}, \ldots, f_{d}\right\}$ is contained in the linear span of $\left\{b_{1}, \ldots, b_{\ell}\right\}$;

(2) the $\ell$ columns of the matrix $\left(\lambda_{j k}\right)$ in the expression

$$
\left(f_{1}, \ldots, f_{d}\right)^{t}=\left(\lambda_{j k}\right)\left(b_{1}, \ldots, b_{\ell}\right)^{t}
$$

are not simultaneously approximable with exponent $s$.

Definitions similar to the two above have been used in [HP] for the study of $C^{\infty}$ hypoellipticity. The simplest such condition was first used by Greenfield and Wallach in [GW], where they showed that $\partial_{t}-\alpha \partial_{x}$ is globally hypoelliptic on $\mathbb{T}^{2}$ if and only if $\alpha$ is not a Liouville number. Also, the notion of a vector satisfying a Diophantine condition was used by Herman $[\mathrm{H}]$ and Yoccoz $[\mathrm{Y}]$.

Next we state our result.

Theorem 1. In $\mathbb{T}^{m+n}$ with variables $(t, x)=\left(t_{1}, \ldots, t_{m}, x_{1}, \ldots, x_{m}\right)$ let the operator

$$
P=-\Delta_{t}-\left(a_{1}(t) \frac{\partial}{\partial x_{1}}+\cdots+a_{n}(t) \frac{\partial}{\partial x_{n}}\right)^{2},
$$

where $a_{j}, j=1, \ldots n$, are real-valued and real-analytic functions defined on $\mathbb{T}^{m}$. Then the operator $P$ is globally $G^{s}$ hypoelliptic on $\mathbb{T}^{m+n}$ if and only if, after a possible renaming of the variables $x_{1}, \ldots, x_{n}$ and the corresponding coefficients $a_{1}, \ldots, a_{n}$, the following Diophantine condition $(D C)_{j}$ holds for some $j \in\{0,1, \ldots$, $n-1\}$.

$(D C)_{j}$

$$
\begin{gathered}
a_{1}, \ldots, a_{n-j} \text { are independent over } \mathbb{R} \text { and } \\
\left(a_{n-j+1}, \ldots, a_{n}\right) \in(S A)_{s}^{c}\left(a_{1}, \ldots, a_{n-j}\right) .
\end{gathered}
$$


For results on the interesting open problems of local and global, analytic and Gevrey hypoellipticity of sublaplacians we refer the reader to the following works as well as the references listed therein: Baouendi and Goulaouic [BG], Bove and Tartakoff [BT1], [BT2], Christ [C1], [C2], [C3], Hanges and Himonas [HH1], [HH2], [HH3], Helffer [H], Metivier [M1], [M2], Pham The Lai and Robert PR], Sjöstrand [S], Tartakoff [T1], T2], T3], and Treves Tr1], Tr2]. While, for some results on hypoellipticity we refer to Hörmander [Ho], Kohn [K], Oleinik and Radkevic [OR], and Rothschild and Stein [RS].

\section{Proof of the theorem}

We first prove the necessity of the condition in the theorem. Assume that the Diophantine condition $(D C)_{j}$ does not hold for any $j \in\{0,1, \ldots, n-1\}$. Then, after a possible renaming of the variables $x_{1}, \ldots, x_{n}$, we must have that either $a_{1}$ is identically equal to zero, or for some $j \in\{0,1, \ldots, n-1\}$ the coefficients $a_{1}, \ldots, a_{n-j}$ are linearly independent, $\left\{a_{n-j+1}, \ldots, a_{n}\right\} \subset \operatorname{span}\left\{a_{1}, \ldots, a_{n-j}\right\}$, and the $(n-j)$ column vectors of the matrix $\left(\lambda_{\ell k}\right)$ in the expression

$$
\left(a_{n-j+1}, \ldots, a_{n}\right)^{t}=\left(\lambda_{\ell k}\right)\left(a_{1}, \ldots, a_{n-j}\right)^{t}
$$

are simultaneously approximable with exponent $s$. In the case that $a_{1}=0$, any function $u=u\left(x_{1}\right)$ not belonging to $G^{s}(\mathbb{T})$ is a solution to $P u=0$, and therefore $P$ is not globally $G^{s}$ hypoelliptic in $\mathbb{T}^{m+n}$. In the second case we have

$$
a_{\ell}=\sum_{k=1}^{n-j} \lambda_{\ell, k} a_{k}, \ell=n-j+1, \ldots, n,
$$

where the vectors $\lambda_{k}=\left(\lambda_{n-j+1, k}, \ldots, \lambda_{n, k}\right), k=1, \ldots, n-j$, are simultaneously approximable with exponent $s$. Then, the operator $P$ takes the form

$$
P=-\Delta_{t}-\left(\sum_{k=1}^{n-j} a_{k}(t)\left[\frac{\partial}{\partial x_{k}}+\sum_{\ell=n-j+1}^{n} \lambda_{\ell, k} \frac{\partial}{\partial x_{\ell}}\right]\right)^{2} .
$$

Since $\lambda_{1}, \ldots, \lambda_{n-j}$ are simultaneously approximable with exponent $s$ there exist $\varepsilon>0$, a sequence $\left\{\xi_{\nu}^{\prime}\right\}=\left\{\left(\xi_{\nu, 1}, \ldots, \xi_{\nu, n-j}\right)\right\} \subset \mathbb{Z}^{n-j}$, and another sequence $\left\{\xi_{\nu}^{\prime \prime}\right\}=$ $\left\{\left(\xi_{\nu, n-j+1}^{\prime \prime}, \ldots, \xi_{\nu, n}^{\prime \prime}\right)\right\} \subset \mathbb{Z}^{j}-\{0\}$ such that $\left|\xi_{\nu}^{\prime \prime}\right| \rightarrow \infty$ as $\nu \rightarrow \infty$ and

$$
\left|\xi_{\nu, k}^{\prime}+\lambda_{k} \cdot \xi_{\nu}^{\prime \prime}\right| \leq e^{-\varepsilon\left|\xi_{\nu}^{\prime \prime}\right|^{\frac{1}{s}}}, k=1, \ldots, n-j, \text { and } \nu=1,2, \ldots
$$

Choosing

$$
u(t, x)=\sum_{\nu=1}^{\infty} e^{i\left(\xi_{\nu}^{\prime} \cdot x^{\prime}-\xi_{\nu}^{\prime \prime} \cdot x^{\prime \prime}\right)}
$$

where $x^{\prime}=\left(x_{1}, \ldots, x_{n-j}\right)$ and $x^{\prime \prime}=\left(x_{n-j+1}, \ldots, x_{n}\right)$, we see that $u \in \mathcal{D}^{\prime}\left(\mathbb{T}^{m+n}\right)-$ $G^{s}\left(\mathbb{T}^{m+n}\right)$, while

$$
P u=\sum_{\nu=1}^{\infty}\left[\sum_{k=1}^{n-j} a_{k}(t)\left(\xi_{\nu, k}^{\prime}-\lambda_{k} \cdot \xi_{\nu}^{\prime \prime}\right)\right]^{2} e^{i\left(\xi_{\nu}^{\prime} \cdot x^{\prime}-\xi_{\nu}^{\prime \prime} \cdot x^{\prime \prime}\right)}
$$

is in $G^{s}\left(\mathbb{T}^{m+n}\right)$ because of inequality (2.1).

To prove the sufficiency of the condition, let $u \in \mathcal{D}^{\prime}\left(\mathbb{T}^{m+n}\right)$ with

$$
P u=f, f \in G^{s}\left(\mathbb{T}^{m+n}\right) .
$$


We will show that $u \in G^{s}\left(\mathbb{T}^{m+n}\right)$ if condition $(D C)_{j}$ holds for some $j \in\{0,1, \ldots$, $n-1\}$. Taking partial Fourier transform with respect to $x$ in (2.2) gives

$$
\left(-\Delta_{t}+\left[a_{1}(t) \xi_{1}+\cdots+a_{n}(t) \xi_{n}\right]^{2}\right) \hat{u}(t, \xi)=\hat{f}(t, \xi) .
$$

For simplicity, we shall assume that we have only one $t$, i.e. $\Delta_{t}=\partial_{t}^{2}$. Multiplying (2.3) by $\overline{\hat{u}}$ and integrating by parts gives

$$
\|\hat{u}(\cdot, \xi)\|_{w}^{2}=\int_{\mathbb{T}} \hat{f}(t, \xi) \overline{\hat{u}}(t, \xi) d t
$$

where for $\varphi \in C^{\infty}(\mathbb{T})$

$$
\|\varphi\|_{w}^{2}=\left\|\varphi_{t}\right\|_{L^{2}(\mathbb{T})}^{2}+\int_{\mathbb{T}} w^{2}(t, \xi)|\varphi(t)|^{2} d t
$$

and $w$ is given by

$$
w(t, \xi)=a_{1}(t) \xi_{1}+\cdots+a_{n}(t) \xi_{n}
$$

We shall need the following:

Lemma 2. If for some $j \in\{0,1,2, \ldots, n-1\}$ the Diophantine condition $(D C)_{j}$ holds, then there exist constants $\alpha=\alpha\left(a_{1}, \ldots, a_{n}\right)>0$ and $\delta=\delta\left(a_{1}, \ldots, a_{n}\right)>0$ such that for any $\varepsilon>0$ and any $\xi \in \mathbb{Z}^{n}-0$ we can find an open interval $I_{\xi} \subset \mathbb{T}$ for which

$$
w^{2}(t, \xi) \geq \alpha C_{\varepsilon} e^{-\varepsilon|\xi|^{\frac{1}{s}}}, \forall t \in I_{\xi},\left|I_{\xi}\right|>\delta,
$$

where $C_{\varepsilon}$ is a positive constant depending only on $\varepsilon$.

Before proving this lemma we shall use it to complete the proof of the theorem. Let $\xi \in \mathbb{Z}^{n}-\{0\}$ be fixed. Then for $\varphi \in C^{\infty}(\mathbb{T}), s \in I_{\xi}$ and $t \in \mathbb{T}$, using the fundamental theorem of calculus we obtain

$$
|\varphi(t)|^{2} \lesssim|\varphi(s)|^{2}+\int_{\mathbb{T}}\left|\varphi_{t}(t)\right|^{2} d t .
$$

Integrating first $t \in \mathbb{T}$ and then $s \in I_{\xi}$ gives

$$
\left|I_{\xi}\right|\|\varphi\|_{L^{2}(\mathbb{T})}^{2} \lesssim \int_{I_{\xi}}|\varphi(s)|^{2} d s+\left|I_{\xi}\right|\left\|\varphi_{t}\right\|_{L^{2}(\mathbb{T})}^{2} .
$$

Using (2.5), for any $\varepsilon>0$ we have

$$
\begin{aligned}
\int_{I_{\xi}}|\varphi(s)|^{2} d s & \lesssim \alpha^{-1} C_{\varepsilon}^{-1} e^{\varepsilon|\xi|^{\frac{1}{s}}} \int_{I_{\xi}} w^{2}(s, \xi)|\varphi(s)|^{2} d s \\
& \lesssim \alpha^{-1} C_{\varepsilon}^{-1} e^{\varepsilon|\xi|^{\frac{1}{s}}} \int_{\mathbb{T}} w^{2}(t, \xi)|\varphi(t)|^{2} d t .
\end{aligned}
$$

This together with (2.6) shows that for any $\varepsilon>0$ there is a constant $C_{\varepsilon}>0$ such that

$$
\|\varphi\|_{L^{2}(\mathbb{T})}^{2} \leq C_{\alpha} C_{\varepsilon}^{-1} e^{\varepsilon|\xi|^{\frac{1}{s}}}\|\varphi\|_{w}^{2}, \forall \varphi \in C^{\infty}(\mathbb{T}), \forall \xi \neq 0,
$$

where $C_{a}$ is a positive constant depending only on $a_{1}, \ldots, a_{n}$.

Applying (2.7) with $\varphi$ replaced by the $C^{\infty}$ function $\hat{u}(\cdot, \xi)$ gives

$$
\begin{aligned}
\|\hat{u}(\cdot, \xi)\|_{L^{2}(\mathbb{T})}^{2} & \leq C_{\alpha} C_{\varepsilon}^{-1} e^{\varepsilon|\xi|^{\frac{1}{s}}}\|\hat{u}(\cdot, \xi)\|_{w}^{2} \\
& \leq C_{\alpha} C_{\varepsilon}^{-1} e^{\varepsilon|\xi|^{\frac{1}{s}}} \int_{\mathbb{T}} \hat{f}(t, \xi) \overline{\hat{u}}(t, \xi) d t .
\end{aligned}
$$


Then applying Cauchy-Schwarz's inequality gives

$$
\|\hat{u}(\cdot, \xi)\|_{L^{2}(\mathbb{T})} \leq C_{\alpha} C_{\varepsilon}^{-1} e^{\varepsilon|\xi|^{\frac{1}{s}}}\|\hat{f}(\cdot, \xi)\|_{L^{2}(\mathbb{T})} .
$$

Since $f \in G^{s}\left(\mathbb{T}^{m+n}\right)$ there is $\varepsilon_{0}>0$ such that

$$
\|\hat{f}(\cdot, \xi)\| \leq c_{0} e^{-\varepsilon_{0}|\xi|^{\frac{1}{s}}}
$$

Choosing in (2.8) $\varepsilon=\frac{\varepsilon_{0}}{2}=\varepsilon_{1}$ and using (2.9) we obtain

$$
\|\hat{u}(\cdot, \xi)\|_{L^{2}(\mathbb{T})} \leq C_{1} e^{-\varepsilon_{1}|\xi|^{\frac{1}{s}}}, \xi \neq 0 .
$$

This and the Cauchy-Schwarz inequality give

$$
|\hat{u}(\tau, \xi)| \leq C_{2} e^{-\varepsilon_{1}|\xi|^{\frac{1}{s}}}, \xi \neq 0 .
$$

Inequality (2.10) together with similar microlocal estimates near each elliptic point $(\tau, 0)$ give the estimate

$$
|\hat{u}(\tau, \xi)| \leq C e^{-\varepsilon_{2}|(\tau, \xi)|^{\frac{1}{s}}}, \forall(\tau, \xi) \in \mathbb{Z}^{m+n},
$$

which shows that $u \in G^{s}\left(\mathbb{T}^{m+n}\right)$. The proof of the theorem will be completed once we prove Lemma 2.

Proof of Lemma 2. If for some $j \in\{0,1, \ldots, n-1\}$ the Diophantine condition $(D C)_{j}$ holds, then

$$
w(t, \xi)=\sum_{k=1}^{n-j} a_{k}(t)\left(\xi_{k}+\lambda_{k} \cdot \xi^{\prime \prime}\right),
$$

where $a_{1}, \ldots, a_{n}$ are independent functions over $\mathbb{R}$, and $\lambda_{k}=\left(\lambda_{n-j+1, k}, \ldots, \lambda_{n, k}\right)$, $k=1, \ldots, n-j$, are not simultaneously approximable. If $\xi^{\prime \prime}=0$, then the independence of $a_{1}, \ldots, a_{n-j}$ and the compactness of the unit sphere $S^{n-j-1}$ give

$$
w^{2}(t, \xi)=\left(\sum_{k=1}^{n-j} a_{k}(t) \xi_{k}\right)^{2}=\left|\xi^{\prime}\right|^{2} w^{2}\left(t, \frac{\xi^{\prime}}{\left|\xi^{\prime}\right|}\right) \geq\left|\xi^{\prime}\right|^{2} \alpha,
$$

for all $t$ in an open interval $I_{\xi}$ with $\left|I_{\xi}\right| \geq \delta$ for some $\delta>0$ and independent of $\xi$. If $\xi^{\prime \prime} \neq 0$, then by letting $\gamma_{k}=\xi_{k}+\lambda_{k} \cdot \xi^{\prime \prime}$ we write $w^{2}(t, \xi)$ in the form

$$
w^{2}(t, \xi)=\left(\sum_{k=1}^{n-j} a_{k}(t) \gamma_{k}\right)^{2}=|\gamma|^{2} w^{2}\left(t, \frac{\gamma}{|\gamma|}\right),
$$

where $\gamma=\left(\gamma_{1}, \ldots, \gamma_{n-j}\right)$. Again, using the independence of $a_{1}, \ldots, a_{n-j}$ and the compactness of the unit sphere $S^{n-j-1}$ we obtain

$$
w^{2}(t, \xi) \geq|\gamma|^{2} \alpha, \forall t \in I_{\gamma},\left|I_{\gamma}\right| \geq \delta .
$$

Since the vectors $\lambda_{1}, \ldots, \lambda_{n-j}$ are not simultaneously approximable with exponent $s$ for any $\varepsilon>0$ there is $C_{\varepsilon}>0$ such that for some $k_{0} \in\{1, \ldots, n-j\}$ we have

$$
\left|\gamma_{k_{0}}\right| \geq C_{\varepsilon} e^{-\varepsilon\left|\xi^{\prime \prime}\right| \frac{1}{s}}
$$

Inequalities (2.11) and (2.12) give

$$
w^{2}(t, \xi) \geq \alpha\left|\gamma_{k_{0}}\right|^{2} \geq \alpha C_{\varepsilon} e^{-\varepsilon\left|\xi^{\prime \prime}\right|^{\frac{1}{s}}} \geq \alpha C_{\varepsilon} e^{-\varepsilon|\xi|^{\frac{1}{s}}},
$$

which completes the proof of the lemma. 


\section{ACKNOWLEDGEMENTS}

Part of this work was done when the author was visiting the Departments of Mathematics of the University of Chicago and Princeton University.

\section{REFERENCES}

[BG] M.S. Baouendi and C. Goulaouic, Nonanalytic-hypoellipticity for some degenerate elliptic operators, Bull. AMS 78, (1972), 483-486. MR 45:5567

[BT1] B. E. Bove and D. Tartakoff, Optimal non-isotropic Gevrey exponents for sums of squares of vector fields, Comm. Partial Differential Equations 22, no. 7-8, (1997), 1263-1282. MR 98f:35026

[BT2] B. E. Bove and D. Tartakoff, On a conjecture of Treves: analytic hypoellipticity and Poisson strata, Indiana Univ. Math. J. 47, no. 2, (1998), 401-417. MR 2000d:35022

[C1] M. Christ, Certain sums of squares of vector fields fail to be analytic hypoelliptic, Comm. in PDE 16, No. 10, (1991), 1695-1707. MR 92k:35056

[C2] M. Christ, Global analytic hypoellipticity in the presence of symmetry, Math. Res. Lett. 1, (1994), 559-563. MR 95j:35047

[C3] M. Christ, Intermediate optimal Gevrey exponents occur, Comm. Partial Differential Equations 22 , no. 3-4, (1997), 359-379. MR 98c:35028

[CH] P. D. Cordaro and A. A. Himonas, Global analytic hypoellipticity for a class of degenerate elliptic operators on the torus, Math. Res. Lett. 1, (1994), 501-510. MR 95j:35048

[GW] S. J. Greenfield and N. R. Wallach, Global hypoellipticity and Liouville numbers, Proceedings of the AMS 31, (1972), 112-114. MR 45:5568

[HH1] N. Hanges and A.A. Himonas, Singular solutions for sums of squares of vector fields, Comm. in PDE 16, No. 8 \& 9, (1991), 1503-1511. MR 92i:35031

[HH2] N. Hanges and A.A. Himonas, Analytic hypoellipticity for generalized Baouendi-Goulaouic operators, J. Funct. Anal. 125, No. 1, (1994), 309-325. MR 95j:35049

[HH3] N. Hanges and A.A. Himonas, Non-analytic hypoellipticity in the presence of symplecticity, Proceedings of AMS 126, No. 2, (1998), 405-409. MR 98d:35031

$[\mathrm{H}] \quad$ B. Helffer, Conditions nécessaires d'hypoanalyticité pour des opérateurs invariants à gauche homogènes sur un groupe nilpotent gradué, J.D. Equations 44, (1982), 460-481. MR 84c:35026

[He] R. Herman, Sur le groupe des diffeomorphismes du tore, Ann. Inst. Fourier, Grenoble, vol 23, (1973), 75-86. MR 52:11988

[Ho] L. Hörmander, Hypoelliptic second order differential equations, Acta Math. 119, (1967), 147-171. MR 36:5526

[HP] A. A. Himonas and G. Petronilho, Global hypoellipticity and simultaneous approximability, J. Funct. Anal. 170, (2000), 356-365. CMP 2000:08

$[\mathrm{K}] \quad$ J.J. Kohn, Pseudo-differential operators and hypoellipticity, Proceedings of Symposia in Pure Mathematics XXIII, (1973), 61-70. MR 49:3356

[M1] G. Metivier, Analytic hypoellipticity for operators with multiple characteristics, Comm. Partial Differential Equations 1 (1981), 1-90. MR 82g:35030

[M2] G. Metivier, Une class d'operateurs non hypoelliptiques analytiques, Indiana Univ. Math. J. 29 (1980), 823-860. MR 82a:35029

[OR] O.A. Oleinik and E. V. Radkevic, Second order equations with nonnegative characteristic form, AMS and Plenum Press, (1973). MR 56:16112

[PR] Pham The Lai and D. Robert, Sur un problème aux valeurs propres non linéaire, Israel J. of Math. 36, (1980), 169-186. MR 80b:35132

[R] L. Rodino, Linear partial differential operators in Gevrey spaces, World Scientific, (1993). MR 95c:35001

[RS] L.P. Rothschild and E.M. Stein, Hypoelliptic differential operators and nilpotent groups, Acta Math 137, (1977), 247-320. MR 55:9171

[S] J. Sjöstrand, Analytic wavefront sets and operators with multiple characteristics, Hokkaido Mathematical Journal 12, (1983), 392-433. MR 85e:35022

[T1] D. S. Tartakoff, Local analytic hypoellipticity for $\square_{b}$ on non-degenerate Cauchy-Riemann manifolds, Proc. Nat. Acad. Sci. U.S.A., 75:7, (1978), 3027-3028. MR 80g:58045

[T2] D. S. Tartakoff, On the local real analyticity of solutions to $\square_{b}$ and the $\bar{\partial}$-Neumann problem, Acta Math. 145, (1980), 117-204. MR 81k:35033 
[T3] D. S. Tartakoff, Global (and local) analyticity for second order orerators constructed from rigid vector fields on products of tori, Trans. AMS 348, No 7, (1996), 2577-2583. MR 96i:35018

[Tr1] F. Treves, Analytic hypo-ellipticity of a class of pseudodifferential operators with double characteristics and applications to the $\bar{\partial}$-Neuman problem, Comm. Partial Differential Equations 3 (1978), 475-642. MR 58:11867

[Tr2] F. Treves, Symplectic geometry and analytic hypo-ellipticity, La Pietra 1996 (Florence), Proc. Sympos. Pure Math., 65, Amer. Math. Soc., Providence, RI, (1999), 201-219. MR 2000b:35031

[Y] J. C. Yoccoz, Recent development in dynamics, Proc. of the International Congress of Mathematicians, Zurich, Switzerland, August 1994, Birkhauser, (1995), 246-265. MR 98e:58146

Department of Mathematics, University of Notre Dame, Notre Dame, Indiana 46556

E-mail address: himonas.1@nd.edu 